\title{
Rechtssoziologie in Deutschland
}

Festrede zum Kongress „Transnationalismus in Recht, Staat und Gesellschaft“ der Vereinigung für Recht und Gesellschaft, Bremen, 3. bis 5 März 2010

\section{Thomas Raiser"}

Mein Beitrag ist als Festrede zu dem Thema „Rechtssoziologie in Deutschland“ angekündigt. Ich vermute, Sie fragen sich mit einem etwas zwiespältigen Gefühl, welche Geduld da noch von Ihnen verlangt wird, nach dem anstrengenden Nachmittag und vor dem ersehnten Büffet. Der Begriff der Festrede ist ja auch nicht sonderlich klar und gebräuchlich. Immerhin: ein wissenschaftlicher Vortrag ist offenkundig nicht gemeint, aber doch etwas mehr als nur ein Grußwort. In der Tat werde ich Ihre Aufmerksamkeit für etwa 20 Minuten in Anspruch nehmen.

\section{A. Vorgeschichte der Vereinigung für Rechtssoziologie}

Als Herr Calliess an mich herantrat mit dem Ansinnen, hier aufzutreten, meinte er, es sei wünschenswert, etwas über die Entstehung der Vereinigung für Rechtssoziologie vorzutragen. Die Jüngeren unter Ihnen wüssten darüber kaum etwas. Es könnte auch im Hinblick auf die beabsichtigte Namensänderung der Vereinigung hilfreich sein. Ich sei als Gründungsmitglied dazu der richtige Mann. Ich solle im Anschluss daran aber auch einige Bemerkungen zur gegenwärtigen Lage der Rechtssoziologie anfügen So hoffe ich, damit auf Ihr Interesse zu stoßen und Ihre Geduld nicht ungebührlich zu strapazieren.

Um die Gründung zu verstehen, müssen Sie sich in Gedanken in die 1960er und 1970er Jahre zurückversetzen. Die Rechtssoziologie war, nach ihren Anfängen im ersten Drittel des 20. Jahrhunderts, die mit den Namen von insbesondere Eugen Ehrlich, Max Weber, Hermann Kantorowicz, Arthur Nußbaum und Theodor Geiger verknüpft sind, während des „tausendjährigen“ Reichs in Deutschland buchstäblich ausgemerzt worden. Auch in den ersten 15 Jahren nach dem zweiten Weltkrieg blieb sie zunächst tot. Erst seit etwa 1960 begann sie wieder zu keimen.

Einen wichtigen Anstoß gab Ralf Dabrendorf, damals der junge shooting star der Soziologie in Deutschland. Er warf die Frage auf, was es bedeute, dass „die eine Hälfte der Gesellschaft über die ihr unbekannte andere Hälfte zu urteilen befugt ist“. ${ }^{1}$ Gemeint war, dass die deutschen Juristen fast ausschließlich aus der bürgerli-

* Prof. em. Dr. Thomas Raiser lehrte bis zu seiner Emeritierung im Jahr 2002 Deutsches und Europäisches Unternehmens- und Wirtschaftsrecht, Rechtssoziologie und Bürgerliches an der Humboldt-Universität zu Berlin, an der er nach wie vor aktiv ist. Die Vortragsform wurde beibehalten. Eine englische Fassung des Textes erscheint parallel im German Law Journal - GLJ, 2010, Nr. 04.

1 R. Dabrendorf, Deutsche Richter. Ein Beitrag zur Soziologie der Oberschicht, 1960, wieder abgedruckt in ders., Gesellschaft und Freiheit, München 1961, S. 176 ff; vgl. auch ders., Gesellschaft und Demokratie in Deutschland, München 1965, S. $260 \mathrm{ff}$. 
chen Oberschicht stammen, maßgeblich an der Ausübung der Staatsmacht beteiligt sind und als solche auch über Streitigkeiten und Straftaten von Menschen aus den sozialen Unterschichten urteilen. Die provokative Bemerkung löste eine größere Zahl berufssoziologischer Untersuchungen aus, welche den Tatbestand im Wesentlichen bestätigten. ${ }^{2}$ Nicht in gleicher Weise gelang es allerdings, den dabei untergründig miterhobenen Vorwurf einer Klassenjustiz ${ }^{3}$ zu erhärten. ${ }^{4}$

1964 eröffnete Ernst Hirsch sein noch heute an der Freien Universität Berlin bestehendes Institut für Rechtssoziologie und Rechtstatsachenforschung. ${ }^{5}$ Er war als Jude 1933 aus Deutschland vertrieben worden und dann in der Türkei als Handelsrechtler und Rechtssoziologe zu höchstem Ansehen gelangt. Trotzdem hatte der damals Regierende Bürgermeister Ernst Reuter ihn zur Rückkehr nach Berlin bewegen können. ${ }^{6}$ Seinem Wirken ist unter anderem zu verdanken, dass Manfred Rebbinder, sein damaliger Assistent, die Werke von Eugen Ehrlich wieder bekannt machen konnte. 7

Schon vorher hatte übrigens Johannes Winckelmann begonnen, auch die Werke von Max Weber neu herauszugeben. ${ }^{8}$ In der Fachjurisprudenz erregten die methodenkritischen Schriften von Josef Esser „Grundsatz und Norm in der richterlichen Fortbildung des Privatrechts"9 und „Vorverständnis und Methodenwahl in der Rechtsfindung " 10 Aufsehen und Unruhe.

Aus dem Bereich der Soziologie zu nennen ist an dieser Stelle weiter Helmut Schelsky. Er war durch seine Bücher aus den 1950er Jahren „Wandlungen der deutschen Familie“, „Soziologie der Sexualität“ und „Die skeptische Generation“ berühmt ge-

2 Vgl. statt aller K. Zwingmann, Zur Soziologie des Richters in der Bundesrepublik Deutschland, Berlin 1966; W. Richter, Zur soziologischen Struktur der deutschen Richterschaft, Stuttgart 1968, W. Kaupen, Die Hüter von Recht und Ordnung, Neuwied 1969; W. Kaupen/T. Rasehorn, Die Justiz zwischen Obrigkeitsstaat und Demokratie, Neuwied 1971; W.O. Weyrauch, Zum Gesellschaftsbild des Juristen, Neuwied 1970.

3 Dazu aus der Weimarer Zeit insbesondere E. Fraenkel, Zur Soziologie der Klassenjustiz, Berlin 1927; aus der Zeit der Bundesrepublik R. Geffken, Klassenjustiz, Frankfurt am Main 1972; Aufsätze von Rasehorn, Wassermann, Kaupen, Lautmann, Sack, u.a. in: Klassenjustiz heute, Vorgänge Heft 1, 1973.

4 Vgl. H. Rottleuthner, Richterliches Handeln, Frankfurt am Main 1973; S. 183 ff; ders., Abschied von der Justizforschung? Für eine Justizforschung mit mehr Recht, in: ZfRSoz 1982, S. 82 ff; T. Raiser, Zum Problem der Klassenjustiz, in: L.M. Friedman/M. Rehbinder (Hrsg.), Zur Soziologie des Gerichtsverfahrens, Jahrbuch für Rechtssoziologie und Rechtstheorie Bd. 4, 1976, S. 123 ff; ders., Rechtssoziologie, Frankfurt am Main 1987, S. 284 ff; K.F. Röhl, Rechtssoziologie, Köln 1987, S. 357 ff.

5 Vgl. E.E. Hirsch, Das Recht im sozialen Ordnungsgefüge, Berlin 1966.

6 Vgl. Hirschs Autobiographie „Als Rechtsgelehrter im Lande Atatürks“, Berlin 2008.

7 Vgl. M. Rehbinder (Hrsg.), Eugen Ehrlich, Recht und Leben, Gesammelte Schriften zur Rechtstatsachenforschung und zur Freirechtslehre, Berlin 1976, ders. (Hrsg.), Eugen Ehrlich, Politische Schriften, Berlin 2007; ferner Rehbinders Buch Die Begründung der Rechtssoziologie durch Eugen Ehrlich, Berlin 1967.

8 Insbesondere M. Weber, Wirtschaft und Gesellschaft, 14. Aufl., Tübingen 1956.

9 Erschienen 1956.

10 1970. 2. Aufl., 1972. 
worden war. Nun wandte er sich der Rechtssoziologie zu und veröffentlichte dazu eine Reihe wichtiger Abhandlungen. ${ }^{11}$ Schon seit Mitte der 1960er Jahre war ferner Niklas Lubmann mit seinen frühen Werken „Grundrechte als Institution“,12 „Legitimation durch Verfahren “13 und dann mit seiner Rechtssoziologie von $1972^{14}$ als leuchtender Stern am Himmel der Rechtssoziologie aufgegangen.

Wer über die deutschen Grenzen hinausblickte, konnte schließlich erkennen, dass sich die Rechtssoziologie auch im Ausland neu hervortat und organisierte. ${ }^{15} 1962$ wurde das Research Committee on Sociology of Law der Internationalen soziologischen Vereinigung gegründet, 1964 die US-amerikanische Law \& Society Association.

\section{B. Die Gründung der Vereinigung für Rechtssoziologie}

Es war für die Rechtssoziologie also eine ungewöhnlich fruchtbare Zeit. Kein Wunder, dass sie auch viele jüngere Wissenschaftler sowohl in der Jurisprudenz als auch in der Soziologie anzog. Vorangetrieben und verstärkt wurde die Anziehungskraft dann aber besonders durch den allgemeinen geistigen Aufbruch, den die Studentenunruhen seit 1967 ausgelöst hatten. Zwar gingen die wenigsten von uns der verblasenen Ideologie ihrer Wortführer auf den Leim. Und wir gingen auch nicht auf die Straße. Aber soziologisches Denken erschien vielen als das geeignete Mittel, die als verknöchert empfundenen Strukturen der herkömmlichen Schuljurisprudenz und der Hierarchie an den Hochschulen aufzubrechen. Die Aufbruchstimmung erhielt dann noch neue Nahrung, als Bundeskanzler Willy Brandt die Parole ausgab: „mehr Demokratie wagen“.

Die Bewegung veranlasste 1972 eine grundlegende Reform der Juristenausbildung. In der neuen, auch in Bremen aufgebauten, einphasigen Ausbildung spielte die soziale Dimension des Rechts eine herausragende Rolle. Aber auch für die herkömmliche zweiphasige Ausbildung schrieben $\$ 5$ a des deutschen Richtergesetzes und die Justizausbildungsordnungen der Bundesländer nunmehr vor, „die gesellschaftlichen Grundlagen des Rechts“ mit zu lehren- und zu prüfen.

Das war für die juristischen Fakultäten und für deren jüngere Mitglieder, die für die Lehre in diesem Bereich in Betracht kamen, eine Herausforderung, auf die sie überhaupt nicht vorbereitet waren. Es entstand der Bedarf, sich über die Lehrinhalte des

11 H. Schelskys Aufsätze zur Rechtssoziologie sind gesammelt in dem Band „Die Soziologen und das Recht“, Opladen 1980; vgl. dazu T. Raiser, Grundlagen der Rechtsoziologie, 5. Aufl., Tübingen 2009 S. $148 \mathrm{ff}$.

12 1967, 4. Aufl. 1999.

13 1969, 6 Aufl. 2001.

14 3. Aufl.1987.

15 Übersicht über die Entwicklung bei T. Raiser, Grundlagen (Fn. 11), S. 41 ff; ferner ausführlicher schon T. Raiser, Die Entstehung der Vereinigung für Rechtssoziologie, in: Soziologie des Rechts, Festschrift für E. Blankenburg, Baden-Baden 1998, S. $11 \mathrm{ff.}$ 
neuen Fachs zu verständigen. Das war 1975 der Anlass für eine Arbeitstagung in Gießen zum Thema „Probleme des rechtssoziologischen Unterrichts für Studenten der Rechtswissenschaft", an der immerhin 35 Fachvertreter, überwiegend Juristen, teilnahmen, unter ihnen auch Josef Esser und Helmut Schelsky. Die Gruppe war, wie nicht anders zu erwarten, nach sozialem und wissenschaftlichem Hintergrund ebenso wie nach der politischen Ausrichtung sehr inhomogen. Gleichwohl wurde im Verlauf der Tagung aber ganz ungeplant der Wunsch laut, den Kreis als Verein zu institutionalisieren. Es wurde eine Kommission gebildet mit dem Auftrag, eine Satzung auszuarbeiten, dem Frau Limbach sowie die Herren Blankenburg, Esser, Hassemer, Heldrich, Lautmann, Raiser, Schelsky und Weiss angehörten, also bis heute die frühen Bannerträger der Rechtssoziologie in Deutschland. Allerdings distanzierte sich Schelsky aus politischen Gründen bald wieder von dem Vorhaben. Esser erklärte sich mit dem wunderbaren Ausspruch zur Mitwirkung bereit: „wenn Sie mich als Covergirl brauchen“.

Auf der Kommissionssitzung in Bielefeld im Herbst 1975 einigte man sich dann nach heftigen Diskussionen auf die Formulierung, die künftige Vereinigung fördere

- die Rechtssoziologie in Forschung und Lehre

- die Zusammenarbeit von Sozialwissenschaftlern und Juristen

- die Integration sozialwissenschaftlicher Erkenntnis und Methoden in Rechtswissenschaft und Rechtspraxis, und

- das Verständnis für rechtliche Entscheidungsprobleme in der sozialwissenschaftlichen Forschung

Die Formulierung reflektierte also bereits damals das klare Bewusstsein für die methodischen und sachlichen Schwierigkeiten, welche Soziologen und Juristen miteinander hatten. In der Gründungsversammlung wurde dann allerdings der erste Satz nach heftigen Diskussionen als überflüssig wieder gestrichen. Im Übrigen gilt, wie Sie wissen, diese Zweckbestimmung bis heute.

Schwierigkeiten bereitete die Formulierung der Voraussetzungen für den Erwerb der Mitgliedschaft. Denn es galt, einerseits die Vereinigung für möglichst viele Interessierte zu öffnen, andererseits aber zu verhindern, dass sie wissenschaftlich nicht ausreichend qualifizierte oder politisch allzu exponierte Bewerber aufnahm und sich dadurch von vornherein kompromittierte, vor allem gegenüber den juristischen Fakultäten. Der Kommissionsvorschlag dazu lautete. „Die Mitgliedschaft kann erwerben, wer auf dem Gebiet der Rechtssoziologie wissenschaftlich publiziert hat. Über den Antrag auf Mitgliedschaft entscheidet der Vorstand mit der Mehrheit von zwei Dritteln seiner Mitglieder. Bei Meinungsverschiedenheiten im Vorstand entscheidet die Mitgliederversammlung“. Doch wurde auch ein Alternativvorschlag formuliert mit dem Wortlaut: „Die Mitgliedschaft kann erwerben, wer auf dem Gebiet der Rechtssoziologie wissenschaftlich publiziert hat. Vom Erfordernis der Promotion 
kann abgesehen werden, wenn rechtssoziologische Arbeiten vorliegen, die einer Dissertation gleichwertig sind“.

Nachdem sich die politische Szene kurze Zeit später wieder beruhigt hatte, erwies sich die Sorge dann glücklicherweise als unberechtigt. Schon auf der Gründungsversammlung entschied sich die Mehrheit für eine liberalere Regelung und begnügte sich mit dem - noch heute geltenden - Satz: „Die Mitgliedschaft kann erwerben, wer die Zwecke der Vereinigung in Forschung oder Lehre vertritt“.

Auf einer Versammlung in Berlin im April 1976 wurde die Gründung formell beschlossen. Immerhin fast 50 Personen nahmen daran teil. Frau Limbach übernahm es, die Eintragung in das Vereinsregister zu besorgen, weshalb die Vereinigung bis heute ihren formellen Sitz in Berlin hat. In den ersten Jahren konnte die Vereinigung dann einen bemerkenswerten Erfolg verzeichnen. Bereits 1977 vermeldete sie 85 Mitglieder, darunter 53 Professoren, 25 sonstige Universitätsangehörige und 10 Praktiker (bei drei Doppelzählungen). 1980 war die Mitgliederzahl auf 140 angewachsen. Das Kind hat sich also als lebenskräftig erwiesen und ist bis heute, mit fast 35 Jahren, erwachsen geworden.

\section{Bemerkungen zur Entwicklung der Vereinigung zwischen $\mathbf{1 9 8 0}$ und 2010}

Allerdings ist auch leicht zu erkennen, dass es in dieser Zeit schwierige Durststrecken durchstehen musste und auch gegenwärtig am Hungertuch nagt. Fragt man nach den Ursachen dafür, kommt ein ganzes Bündel von Gründen zusammen. Ich möchte nur vier davon erwähnen, ohne Anspruch auf Vollständigkeit.

1. Äußerlich gesehen ist der wohl wichtigste Grund der ständige Druck auf die juristischen Fakultäten, trotz der Verminderung der Professorenstellen, der in die umgekehrte Richtung weisenden Vermehrung der Studentenzahlen und der Forderungen nach einer Ausdifferenzierung des Unterrichts ein vollständiges Lehrangebot in den dogmatischen Fächern anzubieten. Ich sage nichts Neues, wenn ich feststelle, dass alle Grundlagenfächer unter diesem Druck leiden, besonders aber die Rechtssoziologie, für die immer weniger Stellen bereitgehalten werden, so dass auch der wissenschaftliche Nachwuchs kaum noch Chancen in dem Fach sieht.

2. Man muss aber, um die Zusammenhänge richtig einzuordnen, noch eine Schicht tiefer graben. Das Zurückdrängen der Rechtssoziologie ist auch eine Reaktion der Schuljurisprudenz auf die seit den 1960er Jahren aufgekommene sozialwissenschaftliche Betrachtung des Rechts, durch welche sie auf mannigfache Weise in Frage gestellt wurde. Die alte und traditionsreiche Rechtswissenschaft sah in dem Emporkömmling einen unerwünschten Nebenbuhler. Rechtssoziologie wurde und wird eben vielfach nicht als notwendige sozialwissenschaftliche Ergänzung der normativ- 
dogmatisch verfahrenden Jurisprudenz angesehen, sondern als eine Verfremdung, die sich zu Lasten der geltenden rechtlichen Ordnung auswirkt.

3. Auf der anderen Seite tragen nicht minder auch die Fachsoziologen zu der Entfremdung bei. Nach allem, was man hört und beobachten kann, findet die Rechtssoziologie auch an den gesellschaftswissenschaftlichen Fakultäten gegenwärtig kaum eine Heimstätte, sondern sieht sich neben Fächern wie zum Beispiel Familiensoziologie, Stadtsoziologie, Parteiensoziologie usw. in den Hintergrund gedrängt. Eine Ursache dafür mag die Ubiquität des Rechts in der vom Prinzip des Rechtsstaats geprägten Gesellschaft sein. Wenn spezielle Rechtsvorschriften auch in den Spezialfächern der Gesellschaftswissenschaften überall eine Rolle spielen, ist der Anreiz anscheinend gering, sich prinzipiell mit der Rolle des Rechts in der Gesellschaft auseinanderzusetzen. Hinzukommt die Schwierigkeit für nicht gelernte Juristen, sich in die hoch komplexen Strukturen des Rechts hineinzudenken.

4. Erwähnen möchte ich schließlich, dass eine andere, in den vergangenen Jahren überaus erfolgreiche transdisziplinäre Denkweise mit dazu beiträgt, rechtssoziologische Forschungsansätze in den Hintergrund zu drängen: die ökonomische Analyse des Rechts. Wir leben ja in einer Periode, in der die Ökonomie das Denken der Menschen nicht nur in der Wissenschaft maßgeblich bestimmt. Doch tun sich Wirtschaftswissenschaftler und Juristen anscheinend auch leichter, einen gemeinsamen Nenner zu finden.

Alle solche Kritik sollte allerdings den Blick dafür nicht verstellen, dass die Rechtssoziologie im Reifungsprozess der vergangenen dreißig Jahre auch wichtige Fortschritte gemacht hat. Auch dazu müssen hier einige wenige Stichworte genügen:

1. Dass das Recht nicht nur ein gedankliches System von logisch aufeinander bezogenen Normen darstellt, sondern einen realen Faktor der Ordnung des gesellschaftlichen Lebens, dass es sich also mit anderen Worten nicht in dem „law in the books“ erschöpft, sondern seinem Wesen nach „law in action" ist und als solches auch wissenschaftlich erforscht werden muss, dürfte inzwischen auch bei den Juristen Allgemeingut geworden sein. Wenngleich die Rechtssoziologie als Lehrfach zurückgedrängt wird, ist sozialwissenschaftlich geprägtes Denken inzwischen in einem Ausmaß in die Arbeit am geltenden Recht und de lege ferenda eingedrungen, welches vor dreißig Jahren noch undenkbar war. Mehrere führende Mitglieder der Vereinigung wurden als Richter an das Bundesverfassungsgericht berufen. ${ }^{16}$ Eine gewisse Festigkeit und Sichtbarkeit hat die Rechtssoziologie auch in Gestalt der inzwischen erschienenen Lehrbücher erlangt. ${ }^{17}$ So gesehen gilt es also zwar, die Basis der Diszi-

16 Frau Limbach als Präsidentin sowie die Herren Hassemer, Hoffmann-Riem und Bryde.

17 Vgl. N. Lubmann, Rechtssoziologie, Hamburg 1972; ders., Das Recht der Gesellschaft, Frankfurt am Main 1993; M. Rehbinder, Rechtssoziologie, 7. Aufl., München 2009; Röhl, Rechtssoziologie (Fn.4); H. Rottleuthner, Einführung in die Rechtssoziologie, Darmstadt 1987; Raiser, Grundlagen (Fn. 11). 
plin als selbständiges Fach in der Forschung und im Lehrbetrieb zu verbreitern, nicht mehr jedoch, ihre Wichtigkeit als solche zu belegen und propagieren.

2. Ungeachtet der erwähnten Engpässe konnten auch in den letzten drei Dezennien wichtige rechtssoziologisch-empirische Forschungen durchgeführt werden. Sie bezogen sich, dank der Initiative und Förderung durch das Bundesministerium der Justiz, großenteils auf Fragen, welche mit der Justizorganisation und der Rechtspflege zu tun hatten. ${ }^{18}$ Vielen von ihnen ist auch ein sichtbarer Erfolg beschieden gewesen, indem sie die Reformgesetzgebung beeinflusst haben.

3. Blickt man auf die gegenwärtigen Anstrengungen sozialwissenschaftlicher Theorie, so fällt auf, dass sich rechtsphilosophisches, rechtssoziologisches und rechtstheoretisches Denken in wachsendem Maß verschränken. Als Beispiel soll nur Jürgen Habermas’ Buch „Faktizität und Geltung“ genannt werden, das schon im Titel die zwei Dimensionen des Rechts zum Ausdruck bringt und im Vorwort alsbald dazu ausführt, Rechtsphilosophie sei längst keine Sache der Philosophen mehr, sondern verlange heute "ein methodenpluralistisches Vorgehen aus der Perspektiven der Rechtstheorie, der Rechtssoziologie und -geschichte, der Moral- und Gesellschaftstheorie". ${ }^{19}$

\section{Ausblick}

Das veranlasst mich, zum Schluss noch einige Bemerkungen anzufügen, wie es nun weiter gehen könnte. Ich erwähne wiederum vier Punkte.

1. Die vorgesehene Namensänderung der Vereinigung halte ich für dringlich. Sie ist geboten, weil die Bezeichnung „Vereinigung für Rechtssoziologie“ von manchen als zu eng, nicht mehr zeitgemäß und daher irreführend angesehen wird. In der Tat muss es heute mehr als vordem darauf ankommen, die Offenheit der Juristen für die Sozialwissenschaften, und zwar für alle, und deren Offenheit für die Fragestellungen und Denkweisen der Jurisprudenz schon im Namen herauszustellen. Auch kommt es darauf an, auf solche Weise die Tore für eine interdisziplinäre Zusammenarbeit so weit wie möglich zu öffnen. Auch die Öffnung gegenüber dem Ausland kommt in der Wendung „Recht und Gesellschaft,, besser zum Ausdruck als in dem dürren Begriff „Rechtssoziologie“, denn dort ist die Wendung „Law and Society“ gebräuchlich.

2. Die interdisziplinäre Zusammenarbeit muss in Zukunft aber auch praktisch verwirklicht oder doch verstärkt werden. Sowohl die theoretischen Konzepte als auch

18 Erwähnt seien statt aller nur H. Rottleuthner/M. Rottleuthner-Lutter, Die Dauer von Gerichtsverfahren, Baden-Baden 1990; R. Wasilewski, Streitverhütung durch Rechtsanwälte, Köln 1990; P, Gilles, Ziviljustiz und Rechtsmittelproblematik, Köln 1992; W. Jagodzinski/T. Raiser/J. Riehl, Rechtsschutzversicherung und Rechtsverfolgung, Köln 1994; J. Stock/P. I. Wolff/H. Thünte, Strukturanalyse der Rechtpflege, Köln 1996.

19 W. Habermas, Faktizität und Geltung, Frankfurt am Main 1992, S. 9. 
die Forschungsmethoden beider Wissenschaftsbereiche haben sich inzwischen in einem Maß ausdifferenziert, dass substantielle Forschung in der Regel nur noch in gemeinsamer Anstrengung mehrerer Vertreter unterschiedlicher Disziplinen erzielt werden können. Nach meiner Beobachtung ist die intellektuelle und soziale Distanz zwischen Gesellschaftswissenschaftlern und Juristen noch immer viel zu groß. Dass es anders sein kann, machen uns die Ökonomen vor, die das Gespräch mit den Wirtschaftsjuristen seit langem und ohne Scheu führen.

3. Je länger desto mehr fällt mir die Kleinteiligkeit der Forschungen zum Thema Recht und Gesellschaft auf. Es gibt eine Vielzahl eng begrenzter Untersuchungen, die nur einen Kreis von Spezialisten interessieren, dem außen stehenden Beobachter dagegen ein eher diffuses Bild von der Relevanz und Leistungsfähigkeit des Forschungsbereichs bieten. Unverkennbar scheint mir auch eine verbreitete Scheu vor tiefer gehenden theoretischen Anstrengungen. Nun ist die Ausdifferenzierung und Hinwendung zu speziellen Themen gewiss auch ein Zeichen der Reife einer Forschungsrichtung. Längerfristig muss eine solche Haltung aber zu einer Zersplitterung führen, welche die Relevanz als eigene Disziplin grundsätzlich in Frage stellt. Deshalb: Wagen Sie sich an anspruchsvollere Projekte heran, deren Ergebnisse dann als Buch erscheinen und Aufsehen erregen können, und zwar sowohl in der Theorie als auch in der Empirie. Nicht immer müssen Veröffentlichungen ja Titel tragen wie „Soziologie vor den Toren der Jurisprudenz" oder Soziologen erstürmen die Zitadelle des Rechts.

4. Als letztes: Die interdisziplinäre und nicht weniger auch die internationale $\mathrm{Zu}$ sammenarbeit bedürfen einer geeigneten und hinreichend einflussreichen Organisation. Ich hätte gewünscht, dass es gelingt, über die bereits vorhandenen Formen der Kooperation hinaus die beiden rechtssoziologischen Vereinigungen zu einem Verband zu verschmelzen. Solange diese neben einander stehen und miteinander konkurrieren, schwächen sie sich gegenseitig. Dritten, zum Beispiel Sponsoren, wird es leicht fallen, sie nach dem Motto „divide et impera“ gegeneinander auszuspielen. Wie viel stärker würde demgegenüber ein Verband auftreten können, in dem alle zusammenwirken, welche die gesellschaftlichen Funktionen des Rechts zu ihrem Forschungsgegenstand machen. Ein solcher Verband könnte nach meiner Überzeugung das gemeinsame Ziel nach innen wie nach außen überzeugender und wirkungsvoller vertreten als das bestehende Nebeneinander. Er könnte zum Beispiel den Plan verfolgen, ein Max-Planck-Institut für die Erforschung von Recht und Gesellschaft ins Leben zu rufen, das der Ort einer wegweisenden und wirkungsmächtigen Forschung zu werden verspricht, insbesondere, solange die Universitäten wegen ihrer herkömmlichen Trennung der Fakultäten dazu nicht in der Lage sind. 